\title{
Normality Assumption Test Using STATCAL (R), SPSS, EViews \& Minitab
}
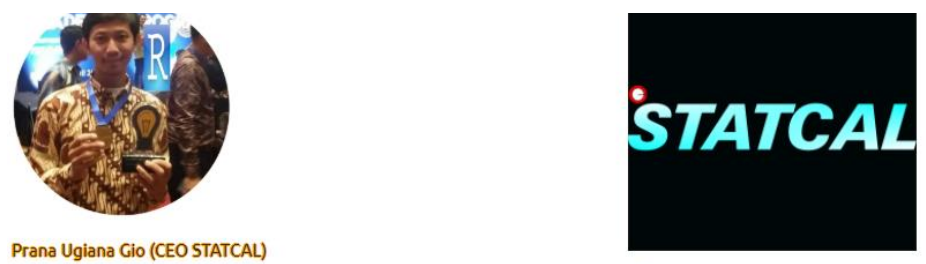

Created by Prana Ugiana Gio \& Rezzy Eko Caraka

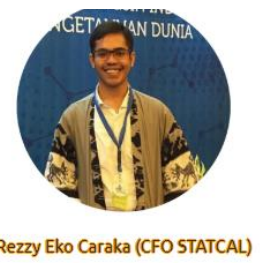

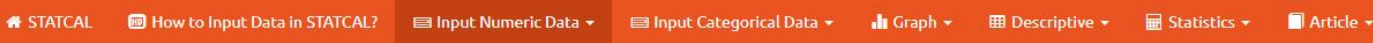

\section{Numeric Data}

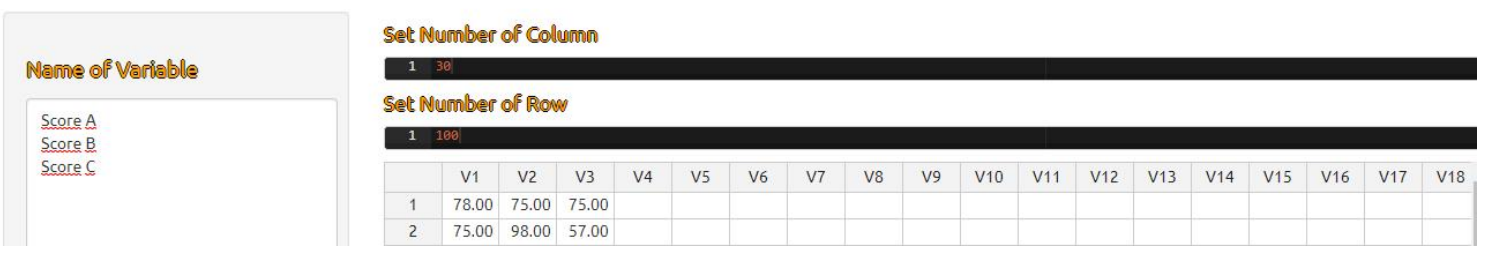

\section{By: Prana Ugiana Gio \& Rezzy Eko Caraka}

\section{Download STATCAL in www.statcal.com}

\section{Citations}

\section{APA}

Gio, P. U., \& Caraka, R. E. (2019, January 23). Normality Assumption Test Using STATCAL (R), SPSS, EViews and Minitab. Retrieved from osf.io/preprints/inarxiv/v96es

\section{MLA}

Gio, Prana U., and Rezzy E. Caraka. "Normality Assumption Test Using STATCAL (R), SPSS, Eviews and Minitab." INA-Rxiv, 23 Jan. 2019. Web.

\section{Chicago}

Gio, Prana U., and Rezzy E. Caraka. 2019. "Normality Assumption Test Using STATCAL (R), SPSS, Eviews and Minitab." INA-Rxiv. January 23. osf.io/preprints/inarxiv/v96es. 


\section{CONTENT}

1.1 Data

1.2 Input Numeric Data in STATCAL

1.3 Normality Assumption Test with STATCAL

1.4 STATCAL's Result

1.5 Comparison with SPSS

1.6 Comparison with EViews

1.7 Comparison with Minitab 
In this article, we will explain step by step how to test normality assumption using STATCAL. Beside that, we will compare STATCAL's result with other software such as SPSS, Minitab and EViews.

\subsection{Data}

Table 1.1.1 is presented data of 10 persons of Score A, B and C.

Table 1.1.1

\begin{tabular}{|c|c|c|c|}
\hline Person & Score A & Score B & Score C \\
\hline 1 & 78 & 75 & 75 \\
\hline 2 & 75 & 98 & 57 \\
\hline 3 & 87 & 46 & 54 \\
\hline 4 & 67 & 88 & 45 \\
\hline 5 & 57 & 65 & 77 \\
\hline 6 & 85 & 45 & 75 \\
\hline 7 & 75 & 78 & 64 \\
\hline 8 & 85 & 75 & 57 \\
\hline 9 & 75 & 75 & 65 \\
\hline 10 & 67 & 75 & 74 \\
\hline
\end{tabular}

Based on the data in Table 1.1.1, we will test normality assumption for Score A, B and C using STATCAL.

\subsection{Input Numeric Data in STATCAL}

Input numeric data in STATCAL as in Figure 1.2.1 until Figure 1.2.3. 


\section{Numeric Data}

Sett Number of Colenno

Sett Nuniber of Row

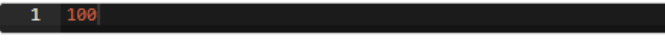

\begin{tabular}{|c|c|c|c|c|c|c|c|c|}
\hline & V1 & V2 & V3 & V4 & V5 & V6 & V7 & V8 \\
\hline 1 & 78.00 & 75.00 & 75.00 & & & & & \\
\hline 2 & 75.00 & 98.00 & 57.00 & & & & & \\
\hline 3 & 87.00 & 46.00 & 54.00 & & & & & \\
\hline 4 & 67.00 & 88.00 & 45.00 & & & & & \\
\hline 5 & 57.00 & 65.00 & 77.00 & & & & & \\
\hline 6 & 85.00 & 45.00 & 75.00 & & & & & \\
\hline 7 & 75.00 & 78.00 & 64.00 & & & & & \\
\hline 8 & 85.00 & 75.00 & 57.00 & & & & & \\
\hline 9 & 75.00 & 75.00 & 65.00 & & & & & \\
\hline 10 & 67.00 & 75.00 & 74.00 & & & & & \\
\hline
\end{tabular}

Figure 1.2.1 Input Numeric Data

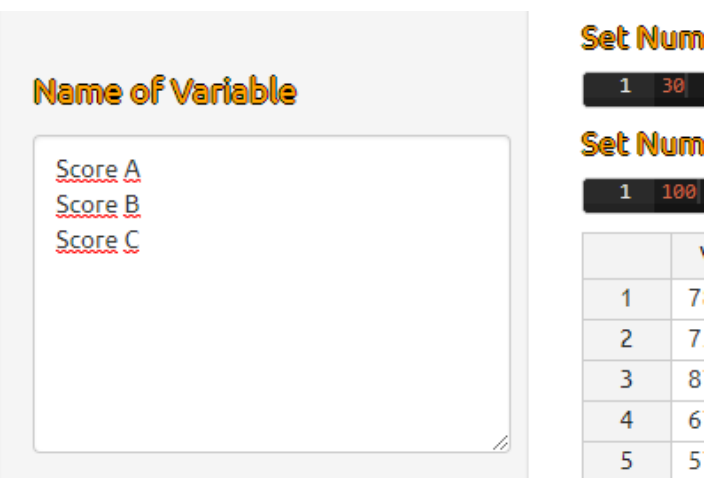

Figure 1.2.2 Giving Name of Each Variable

\section{Your Numeric Data}

\begin{tabular}{lrrr}
\multicolumn{5}{c}{ Score A Score B Score C } \\
1 & 78 & 75 & 75 \\
2 & 75 & 98 & 57 \\
3 & 87 & 46 & 54 \\
4 & 67 & 88 & 45 \\
5 & 57 & 65 & 77 \\
6 & 85 & 45 & 75 \\
7 & 75 & 78 & 64 \\
8 & 85 & 75 & 57 \\
9 & 75 & 75 & 65 \\
10 & 67 & 75 & 74 \\
& & & \\
Number of NA in Score A $=0$ \\
Number of NA in Score B $=0$ \\
Number of NA in Score C $=0$
\end{tabular}

Figure 1.2.3 Your Numeric Data Must be Appeared in "Your Numeric Data" Part 


\subsection{Normality Assumption Test with STATCAL}

For testing normality assumption test in STATCAL, choose Statistics => Test of Normality

(Figure 1.3.1). In Figure 1.3.2, Score A, B and C are moved to the right box.

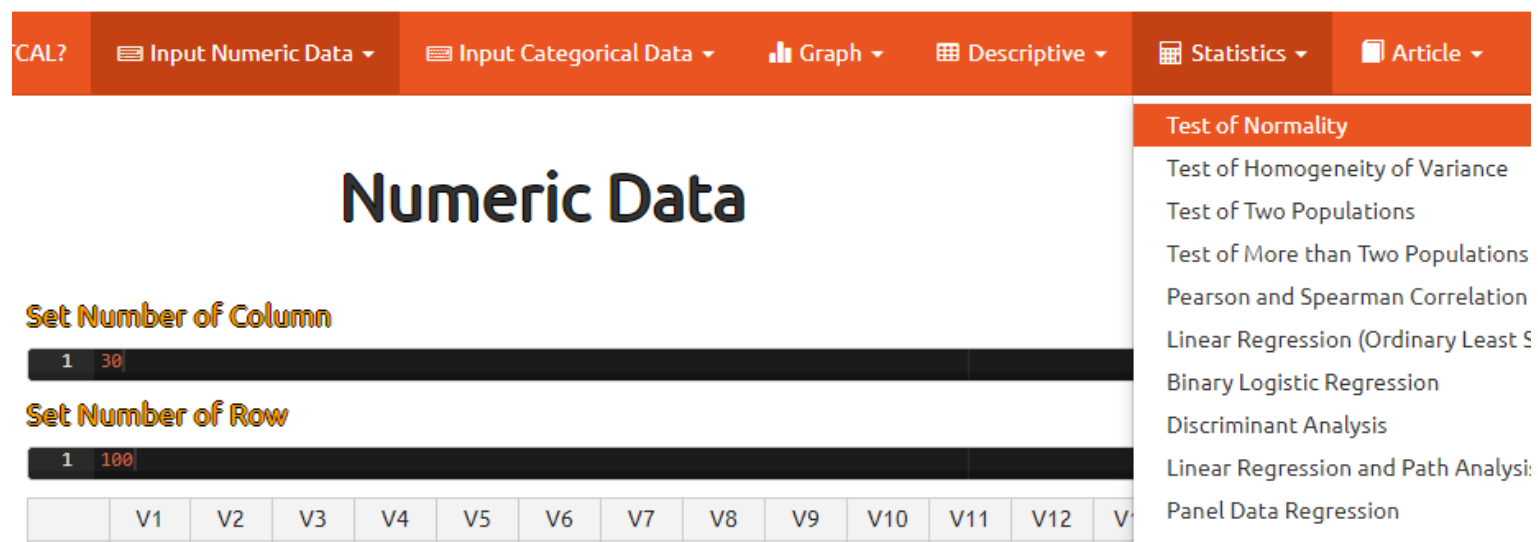

Figure 1.3.1 Normality Assumption Test Menu in STATCAL

Test of Normality (Assumption)

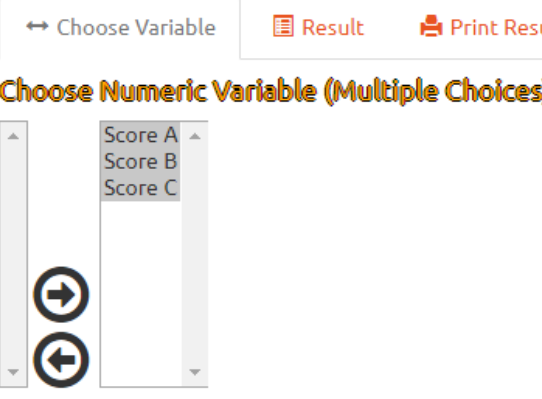

Figure 1.3.2 Selection of Variable

\subsection{STATCAL's Result}

Result of normality assumption test based on STATCAL can be seen in Result part. 


\section{Test of Normality (Assumption)}

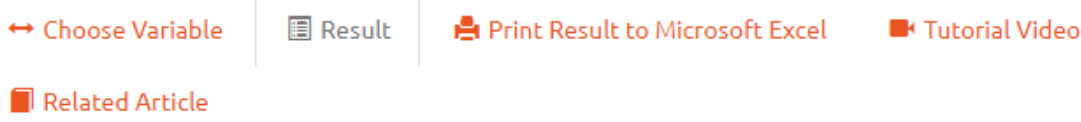

\section{Boxplot}
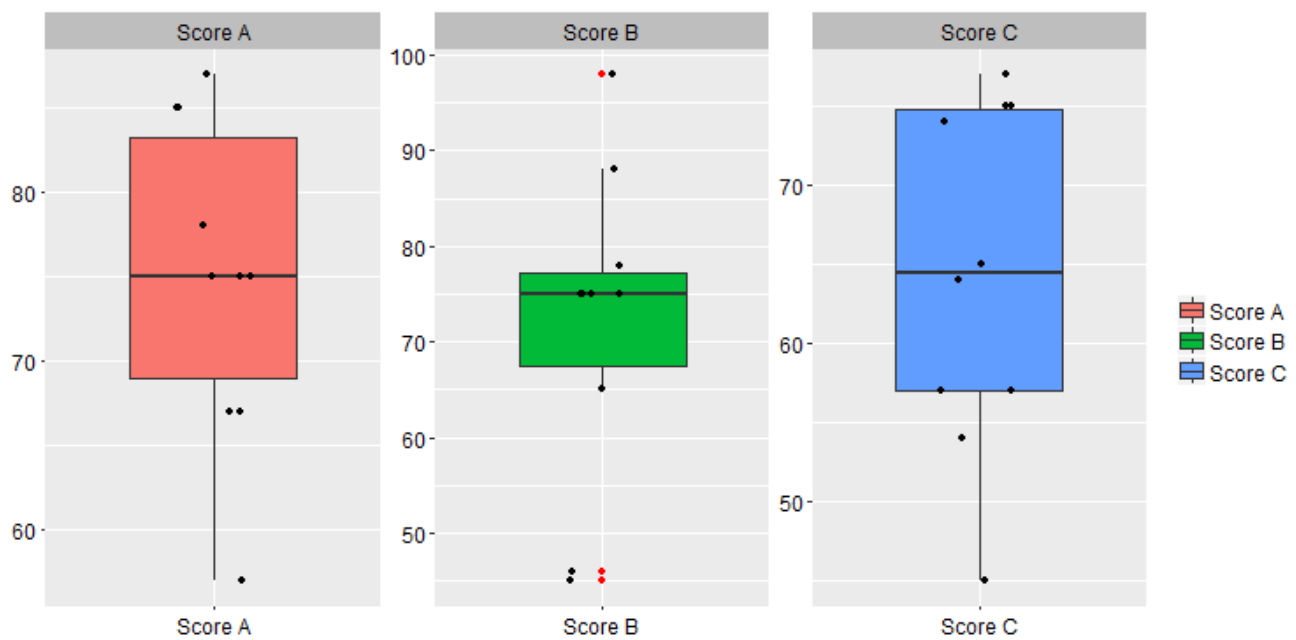

Figure 1.4.1 Boxplot

\section{Kolmogorov-Smirnov Test (Asymptotic Approach)}

\begin{tabular}{|c|c|c|c|c|c|c|}
\hline Show & $10 \mathrm{~V}$ entries & & & Search: & & \\
\hline & Variable $\approx$ & $\begin{array}{r}\text { Statistic of } \\
\text { Kolmogorov-Smirnov } \\
\text { (KS) }\end{array}$ & $\begin{array}{l}\text { P. } \\
\text { Value } \\
\text { of } \mathrm{KS}\end{array}$ & Conclusion & $\hat{\nabla}$ & $n$ \\
\hline 1 & Score A & 0.19578 & 0.83801 & $\begin{array}{l}\mathrm{p} \text {-value }>0.05 \text {, assumption of normality is } \\
\text { received, at the level of significance } 5 \%\end{array}$ & & 10 \\
\hline 2 & Score B & 0.27202 & 0.44993 & $\begin{array}{l}\mathrm{p} \text {-value }>0.05 \text {, assumption of normality is } \\
\text { received, at the level of significance } 5 \%\end{array}$ & & 10 \\
\hline 3 & Score C & 0.21315 & 0.75389 & $\begin{array}{l}\mathrm{p} \text {-value }>0.05 \text {, assumption of normality is } \\
\text { received, at the level of significance } 5 \%\end{array}$ & & 10 \\
\hline
\end{tabular}

Figure 1.4.2 Normality Assumption Test Result Based on

Kolmogorov-Smirnov Test (Asymptotic Approach) 


\section{Kolmogorov-Smirnov Test (Exact Approach)}

Show 10 entries

Search:

\begin{tabular}{|c|c|c|c|c|c|c|}
\hline & Variable & $\begin{array}{r}\text { Statistic of } \\
\text { Kolmogorov-Smirnov } \\
(\mathrm{KS})\end{array}$ & $\begin{array}{l}\text { P- } \\
\text { Value } \\
\text { of KS }\end{array}$ & Conclusion & $\Leftrightarrow$ & $n \neq$ \\
\hline 1 & Score A & 0.19578 & 0.77032 & $\begin{array}{l}\mathrm{p} \text {-value }>0.05 \text {, assumption of normality is } \\
\text { received, at the level of significance } 5 \%\end{array}$ & & 10 \\
\hline 2 & Score B & 0.27202 & 0.38011 & $\begin{array}{l}\mathrm{p} \text {-value }>0.05 \text {, assumption of normality is } \\
\text { received, at the level of significance } 5 \%\end{array}$ & & 10 \\
\hline 3 & Score C & 0.21315 & 0.67945 & $\begin{array}{l}\mathrm{p} \text {-value }>0.05 \text {, assumption of normality is } \\
\text { received, at the level of significance } 5 \%\end{array}$ & & 10 \\
\hline
\end{tabular}

Figure 1.4.3 Normality Assumption Test Result Based on Kolmogorov-Smirnov Test (Exact Approach)

Shapiro-Wilk Test

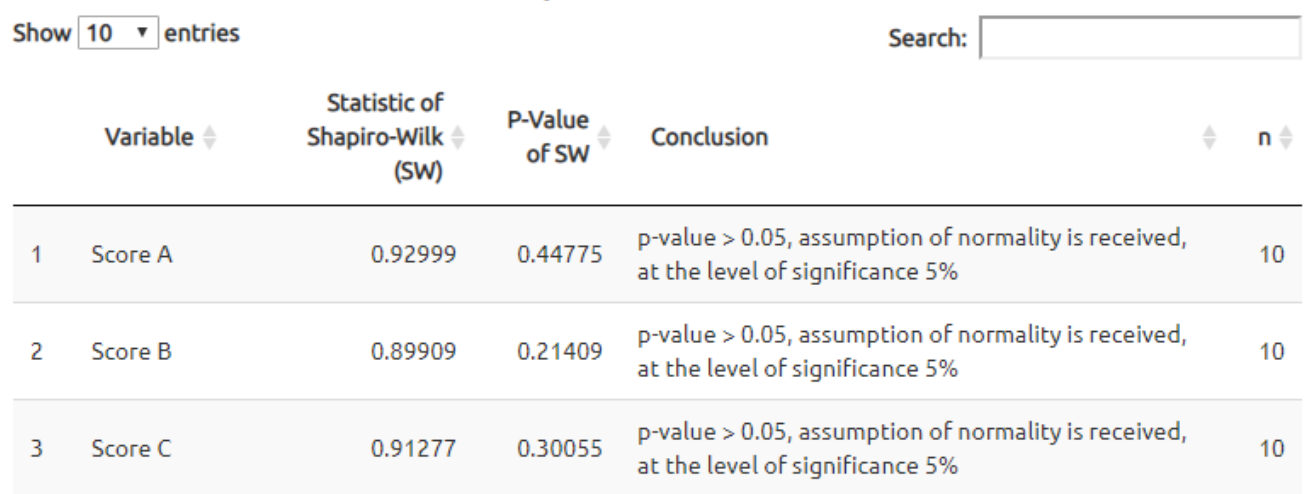

Figure 1.4.4 Normality Assumption Test Result Based on

Shapiro-Wilk Test

Jarque-Bera Test

\begin{tabular}{|c|c|c|c|c|c|}
\hline \multirow[t]{2}{*}{ Show } & $10 \quad$ entries & \multirow[b]{2}{*}{$\begin{array}{r}\text { Statistic of } \\
\text { Jarque-Bera (JB) }\end{array}$} & \multicolumn{3}{|c|}{ Search: } \\
\hline & Variable $\curvearrowright$ & & $\begin{array}{c}\text { P- } \\
\text { Value } \\
\text { of JB }\end{array}$ & Conclusion & 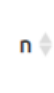 \\
\hline 1 & Score A & 0.50873 & 0.77541 & $\begin{array}{l}p \text {-value }>0.05 \text {, assumption of normality is received, at } \\
\text { the level of significance } 5 \%\end{array}$ & 10 \\
\hline 2 & Score B & 0.37441 & 0.82927 & $\begin{array}{l}\text { p-value }>0.05 \text {, assumption of normality is received, at } \\
\text { the level of significance } 5 \%\end{array}$ & 10 \\
\hline 3 & Score C & 0.69167 & 0.70763 & $\begin{array}{l}p \text {-value }>0.05 \text {, assumption of normality is received, at } \\
\text { the level of significance } 5 \%\end{array}$ & 10 \\
\hline
\end{tabular}

Figure 1.4.5 Normality Assumption Test Result Based on Jarque-Bera Test 


\section{Anderson-Darling Test}

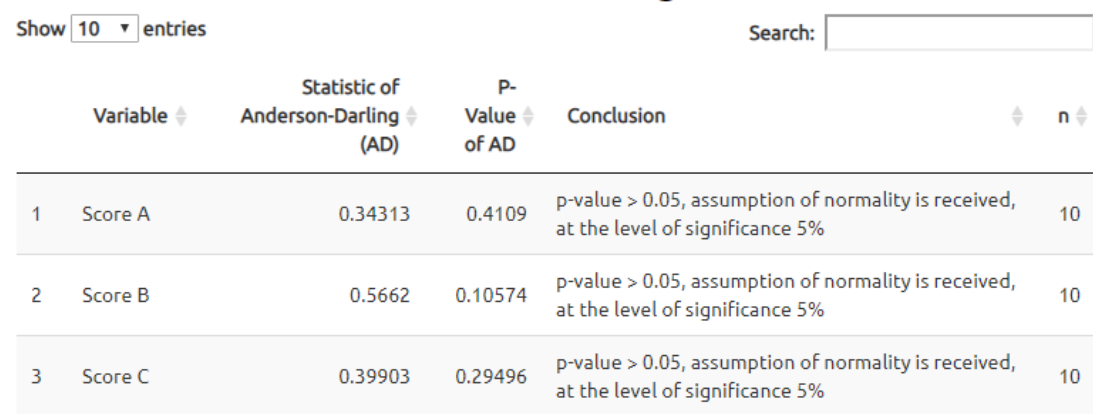

Figure 1.4.6 Normality Assumption Test Result Based on Anderson-Darling Test

\subsection{Comparison with SPSS}

Given SPSS's result for normality assumption test based on Kolmogorov-Smirnov and Shapiro-Wilk test. We use SPSS version 17.

\begin{tabular}{|c|c|c|c|c|}
\hline \multicolumn{5}{|c|}{ 阴 SPSS Data.sav [DataSet0] - SPSS Statistics Data Editor } \\
\hline Eile Edit & View Data I & istorm Ana & Graphs & tillities \\
\hline \multicolumn{5}{|c|}{ 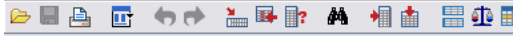 } \\
\hline \multirow[t]{2}{*}{$1:$ ScoreA } & \multicolumn{2}{|c|}{78.0} & & \\
\hline & ScoreA & ScoreB & ScoreC & var \\
\hline 1 & 78.00 & 75.00 & 75.00 & \\
\hline 2 & 75.00 & 98.00 & 57.00 & \\
\hline 3 & 87.00 & 46.00 & 54.00 & \\
\hline 4 & 67.00 & 88.00 & 45.00 & \\
\hline 5 & 57.00 & 65.00 & 77.00 & \\
\hline 6 & 85.00 & 45.00 & 75.00 & \\
\hline 7 & 75.00 & 78.00 & 64.00 & \\
\hline 8 & 85.00 & 75.00 & 57.00 & \\
\hline 9 & 75.00 & 75.00 & 65.00 & \\
\hline 10 & 67.00 & 75.00 & 74.00 & \\
\hline
\end{tabular}

Figure 1.5.1 Data in SPSS

\begin{tabular}{|c|c|c|c|c|}
\hline & & ScoreA & ScoreB & Scorec \\
\hline $\mathrm{N}$ & & 10 & 10 & 10 \\
\hline \multirow[t]{2}{*}{ Normal Parameters ${ }^{\mathrm{a} . . \mathrm{b}}$} & Mean & 75.1000 & 72.0000 & 64.3000 \\
\hline & Std. Deviation & 9.45692 & 16.52607 & 10.90413 \\
\hline \multirow[t]{3}{*}{ Most Extreme Differences } & Absolute & .196 & .272 & .213 \\
\hline & Positive & .104 & .158 & .148 \\
\hline & Negative & -.196 & -.272 & -.213 \\
\hline Kolmogorov-Smirnov $Z$ & & .619 & .860 & .674 \\
\hline Asymp. Sig. (2-tailed) & & .838 & .450 & .754 \\
\hline Exact Sig. (2-tailed) & & .770 & .380 & .679 \\
\hline Point Probability & & .000 & .000 & .000 \\
\hline
\end{tabular}

Figure 1.5.2 Normality Assumption Test Result Based on Kolmogorov-Smirnov Test (Asymptotic \& Exact Approach) 


\begin{tabular}{|c|c|c|c|c|c|c|}
\hline & \multicolumn{3}{|c|}{ Kolmogorov-Smirnov ${ }^{a}$} & \multicolumn{3}{|c|}{ Shapiro-wilk } \\
\hline & Statistic & $\mathrm{df}$ & Siq. & Statistic & $\mathrm{df}$ & Siq. \\
\hline ScoreA & .196 & 10 & $.200^{x}$ & .930 & 10 & .448 \\
\hline ScoreB & .272 & 10 & .035 & .899 & 10 & .214 \\
\hline Scorec & .213 & 10 & $.200^{\pi}$ & .913 & 10 & .301 \\
\hline
\end{tabular}

a. Lilliefors Significance Correction

*. This is a lower bound of the true significance.

\section{Figure 1.5.3 Normality Assumption Test Result Based on}

\section{Shapiro-Wilk Test}

\subsection{Comparison with EViews}

Given EViews's result for normality assumption test based on Jarque-Bera test. We use EViews version 7.

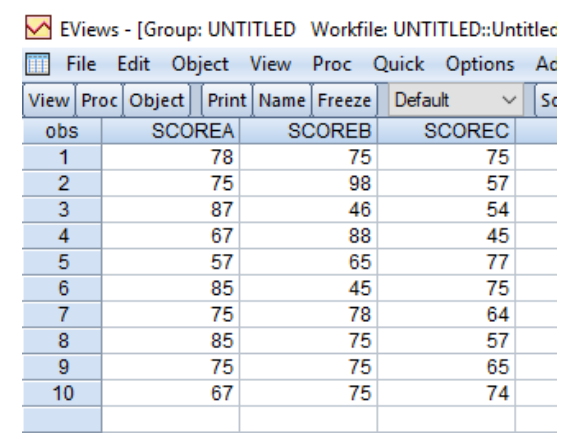

Figure 1.6.1 Data in EViews

\begin{tabular}{|c|c|c|c|c|c|}
\hline \multicolumn{2}{|c|}{ ఐ EViews - [Group: UNTITLED } & \multicolumn{4}{|c|}{ Workfile: UNTITLED::Untitled\] } \\
\hline \multicolumn{2}{|c|}{ 㭵 File Edit Object View } & \multicolumn{4}{|c|}{ Proc Quick Options Add-ins } \\
\hline \begin{tabular}{|l|l|l|} 
View & Proc & Object \\
\end{tabular} & Print Name & Freeze & Sample & Sheet Stats & Spec \\
\hline & SCOREA & $\operatorname{scc}$ & OREB & SCOREC & \\
\hline Mean & 75.10000 & 72. & 00000 & 64.30000 & \\
\hline Median & 75.00000 & 75.0 & 00000 & 64.50000 & \\
\hline Maximum & 87.00000 & $98 . C$ & 00000 & 77.00000 & \\
\hline Minimum & 57.00000 & 45.0 & 00000 & 45.00000 & \\
\hline Std. Dev. & 9.456920 & 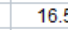 & 52607 & 10.90413 & \\
\hline Skewness & -0.462867 & -0.4 & 04966 & -0.329580 & \\
\hline Kurtosis & 2.396702 & $2.5 \mathrm{c}$ & 07463 & 1.892974 & \\
\hline Jarque-Bera & 0.508729 & 0.37 & 74410 & 0.691666 & \\
\hline Probability & 0.775409 & 0.82 & 29274 & 0.707631 & \\
\hline Sum & 751.0000 & 720 & .0000 & 643.0000 & \\
\hline Sum Sq. Dev. & 804.9000 & 245 & 58.000 & 1070.100 & \\
\hline Observations & 10 & & 10 & 10 & \\
\hline
\end{tabular}

Figure 1.6.2 Normality Assumption Test Result Based on Jarque-Bera Test 


\subsection{Comparison with Minitab}

Given Minitab's result for normality assumption test based on Anderson-Darling test. We use Minitab version 16.

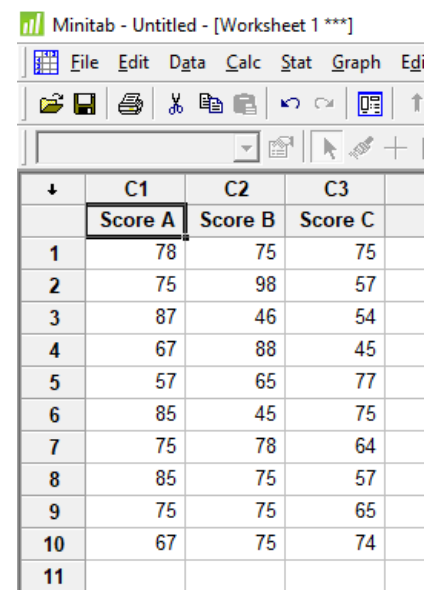

Figure 1.7.1 Data in Minitab

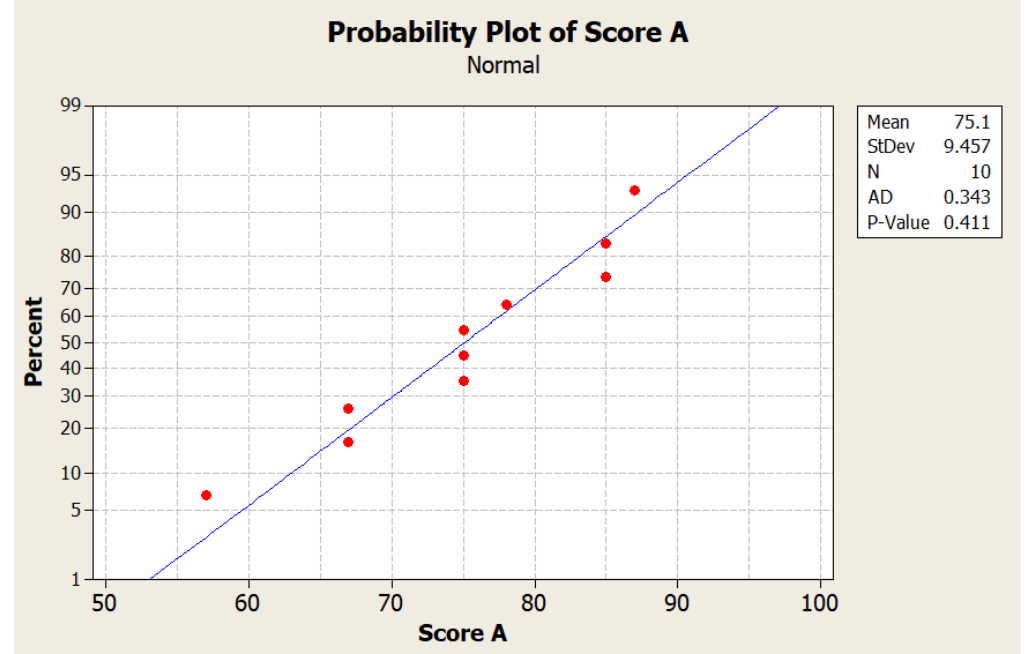

Figure 1.7.2 Normality Assumption Test Result Based on Anderson-Darling Test (Score A) 


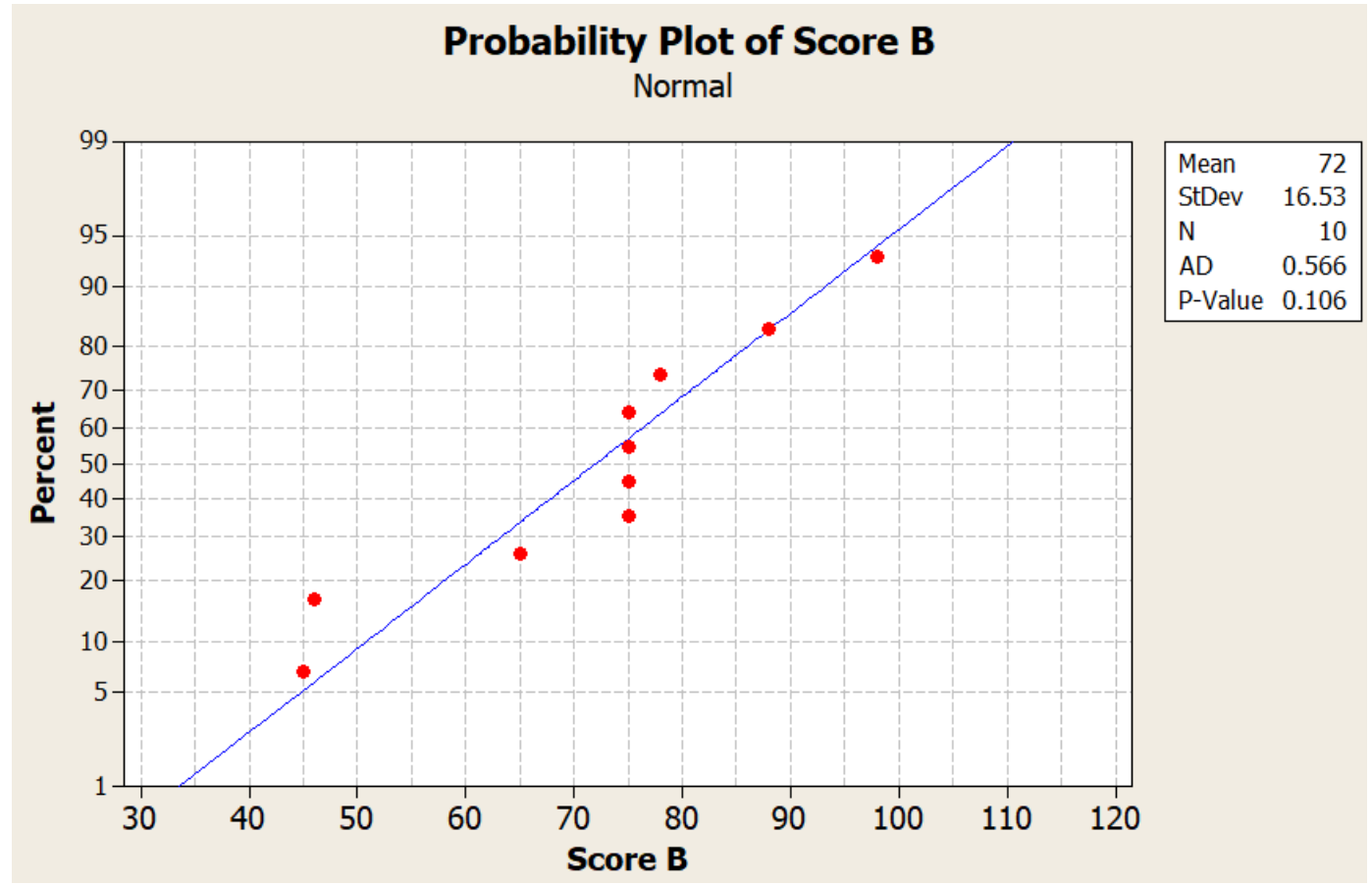

Figure 1.7.3 Normality Assumption Test Result Based on Anderson-Darling Test (Score B)

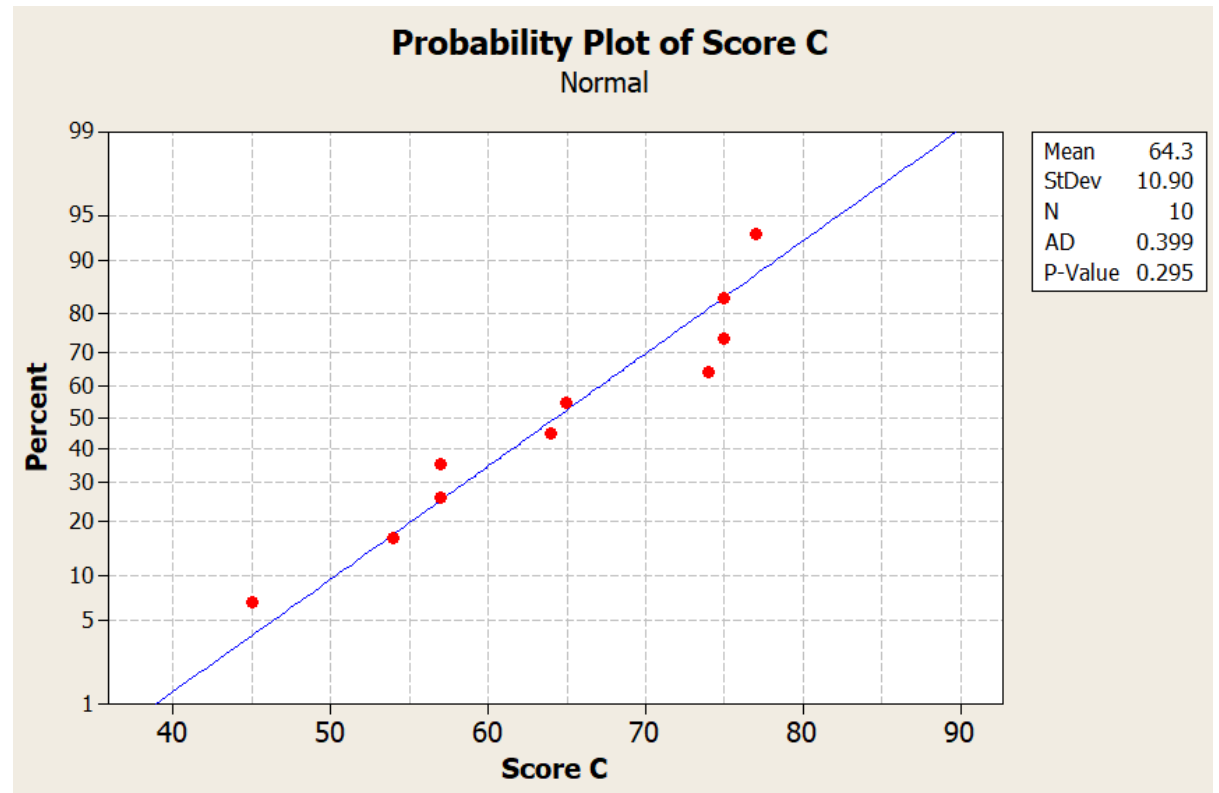

Figure 1.7.4 Normality Assumption Test Result Based on Anderson-Darling Test (Score C) 